\title{
Immune checkpoint inhibitor therapy in biliary tract cancer (cholangiocarcinoma)
}

\author{
Christopher D. Jakubowski, Nilofer S. Azad \\ Bloomberg-Kimmel Institute for Cancer Immunotherapy, Sidney Kimmel Comprehensive Cancer Center, Johns Hopkins University School of \\ Medicine, Baltimore, MD, USA \\ Contributions: (I) Conception and design: All authors; (II) Administrative support: None; (III) Provision of study materials or patients: None; (IV) \\ Collection and assembly of data: None; (V) Data analysis and interpretation: None; (VI) Manuscript writing: All authors; (VII) Final approval of \\ manuscript: All authors. \\ Correspondence to: Nilofer S. Azad. 1450 Orleans Street, Room 4M10, Baltimore, MD 21287, USA. Email: nazad2@jhmi.edu.
}

\begin{abstract}
Biliary tract cancer (BTC) is a rare malignancy with overall poor prognosis. There are limited options regarding systemic therapy for this disease and historically only multi-agent chemotherapy regimens achieve meaningful responses that are often short lived. In the past several years immune checkpoint inhibitor (ICI) therapy has been established as an effective systemic therapy option in many solid tumors. The BTC tumor microenvironment (TME) including immune cells ( $\mathrm{T}$ cells, macrophages, dendritic cells and natural killer cells) and immune checkpoint expression has been characterized. Findings have clinical implications that suggest that this entity is potentially amenable to immunomodulation, including via checkpoint inhibition. Single agent ICI studies have only been reported in the past few years and have mostly targeted the checkpoints PD-1 and PD-L1. As in other tumor subtypes patients with rare mismatch repair deficiency or microsatellite instability appear to have exquisite sensitivity to checkpoint inhibition. Abstracts and published studies suggest modest but real responses in all subtypes including objective response rates (ORRs) in the 5-20\% range and meaningful disease control. They have paved the way for novel combination trials featuring a variety of treatment strategies and agents that look to enhance ICI efficacy and create longterm responders.
\end{abstract}

Keywords: Biliary tract cancer (BTC); cholangiocarcinoma; immunotherapy; immune checkpoint inhibitors (ICIs); tumor microenvironment (TME)

Submitted Jun 30, 2019. Accepted for publication Dec 05, 2019.

doi: $10.21037 /$ cco.2019.12.10

View this article at: http://dx.doi.org/10.21037/cco.2019.12.10

\section{Overview}

Biliary tract cancer (BTC) arises from epithelial cells lining the liver internal bile ducts, external bile ducts and gallbladder. BTCs include intrahepatic cholangiocarcinomas (ICC), extrahepatic cholangiocarcinomas (ECC) and gallbladder carcinomas (GBC) each with distinct molecular and clinical phenotypes but a common origin from biliary epithelium. BTC has an incidence approximated to be 18,000 cases per year in the United States including 6,000 ICC cases and 12,000 gallbladder and other extra hepatic biliary cancer cases (1-4). The only curative modality for this entity is surgical resection in early disease; for unresectable or metastatic BTC, historic 5-year survival rates are daunting at only $10-20 \%(5-8)$. Standard firstline treatment for advanced BTC involves gemcitabine based regimens usually combined with a platinum agent. In the ABC-02 trial that established gemcitabine plus cisplatin as the standard of care, the objective response rate (ORR) of the combination was $26 \%$ compared to $15 \%$ for gemcitabine alone. The median progression free survival (mPFS) and overall survival (mOS) in the gemcitabine plus cisplatin arm was 8.0 and 11.7 months, respectively, the present benchmark for survival in advanced disease (mPFS 
5.0 months and mOS 8.1 months in gemcitabine alone arm) (9). There is no established second line treatment for advanced BTC, but a recent randomized phase III trial (ABC-06) showed a mOS for 5-fluorouracil and oxaliplatin (FOLFOX) of 6.2 months in patients with disease progression after gemcitabine plus cisplatin (10). These outcomes will form the basis of comparison to the trials described in this review.

With limited treatment options and dismal overall prognosis, new therapies and approaches are an urgent unmet need in BTC (11). Clinical trials using targeted agents have historically shown only modest responses in BTC patients, though there has been recent success in treating cholangiocarcinomas with isocitrate dehydrogenase-1 (IDH1) and FGFR genetic alterations with novel targeted agents (12-15). Even in these settings, responses have variable durability and more effective, durable therapies are needed. Immunotherapy has had tremendous success in treating patients with cancers such as melanoma and non-small cell lung cancer, dramatically altering the natural history of these diseases. In BTC, immunotherapy strategies have included autologous cell transfer, vaccinations and immunomodulatory approaches with immune checkpoint inhibitors (ICIs), the latter of which is the largest area of ongoing research. In this review, we describe the rationale for ICI therapy by summarizing the clinically relevant tumor immunology in BTC including the innate and adaptive immune response and immune checkpoint molecule expression in BTC. Subsequently we discuss completed and ongoing clinical trials involving ICI in BTC.

\section{Innate and adaptive immune cells in BTC tumors and their clinical significance}

The immune system plays an important role in the etiology of BTC, and most BTCs are thought to arise from chronic inflammatory states in the biliary tract $(16,17)$. The frequency of tumor infiltration by specific immune cells in ICC, ECC and GBC and their effect on outcomes has been described and summarized (18). Natural killer cells infiltrate ICC, ECC and GBC in the range of $16-21 \%$ of cases but have not been correlated with outcomes. CD4+ and $\mathrm{CD} 8+$ positive $\mathrm{T}$ cells have been shown to infiltrate ICC, ECC and GBC tumors in the $30-51 \%$ range and are associated with reduced probability of metastases and improved survival in ECC. Several studies have shown that increased tumor infiltrating lymphocytes (TILs) (CD4+ helper T-lymphocytes and CD8+ cytotoxic T-lymphocytes) and dendritic cells portend improved outcomes (19-22). Macrophages appear to strongly infiltrate all three subtypes (70-87\% infiltration) and the degree of infiltration is associated with more advanced disease (18).

With clinical disease progression from biliary intraepithelial neoplasia to primary carcinoma to metastasis, there is an increase in innate immune cells including tumor infiltrating macrophages and a decrease in adaptive immune cells. These activated macrophages are believed to promote tumor growth by releasing pro-inflammatory and proangiogenic cytokines (19). A study evaluating 114 ECC tumors showed both depletion of adaptive immune response (CD4+, CD8+, and Foxp3+ T lymphocytes) and enrichment of components in the innate immune response $(\mathrm{CD} 66 \mathrm{~b}+$ tumor associated neutrophils, TANs; and CD163+ M2 tumor-associated macrophages, TAMs) is correlated with poor prognosis, with reduced recurrence free survival (RFS) and chemotherapy resistance (23). The neutrophil-to-lymphocyte ratio (NLR) has shown to be prognostic in cholangiocarcinoma with a higher ratio giving poorer prognosis (24). In a related analysis, another study found that an elevated NLR was associated with decreased percentage of TILs within surgical samples of 102 ICC patients (25).

\section{Immune checkpoint molecules and their clinical implications in BTC}

It has been demonstrated that PD-L1 expression is present in a majority of BTC patient samples at baseline (49-94\%), and is associated with poor survival (26-30). In a study by Gani et al. the expression of PD-L1 within the tumor front was associated with an almost $60 \%$ worse survival (30). Checkpoint expression has been investigated in the BTC subtypes. In a study by Lim et al., a higher ratio of PD-1 positive to CD8+ TILs were associated with poorer OS, recurrence-free survival (RFS), and distant metastasisfree survival in patients with ECC (31). PD-L1 and PD-1 expression has been shown to be upregulated in ICC samples. This upregulation was again associated with worse outcomes and this was possibly due to less CD8+ $\mathrm{T}$ cell expression in the PD-L1 positive tumors (28). The soluble form of PDL1 measured in the serum from 158 advanced BTC patients before initiation of palliative chemotherapy showed high serum PD-L1 was associated with worse OS (32).

Regarding other immune checkpoints studied in BTC, tumors with high $\mathrm{B} 7-\mathrm{H} 4$ and FOXP3 expression in 


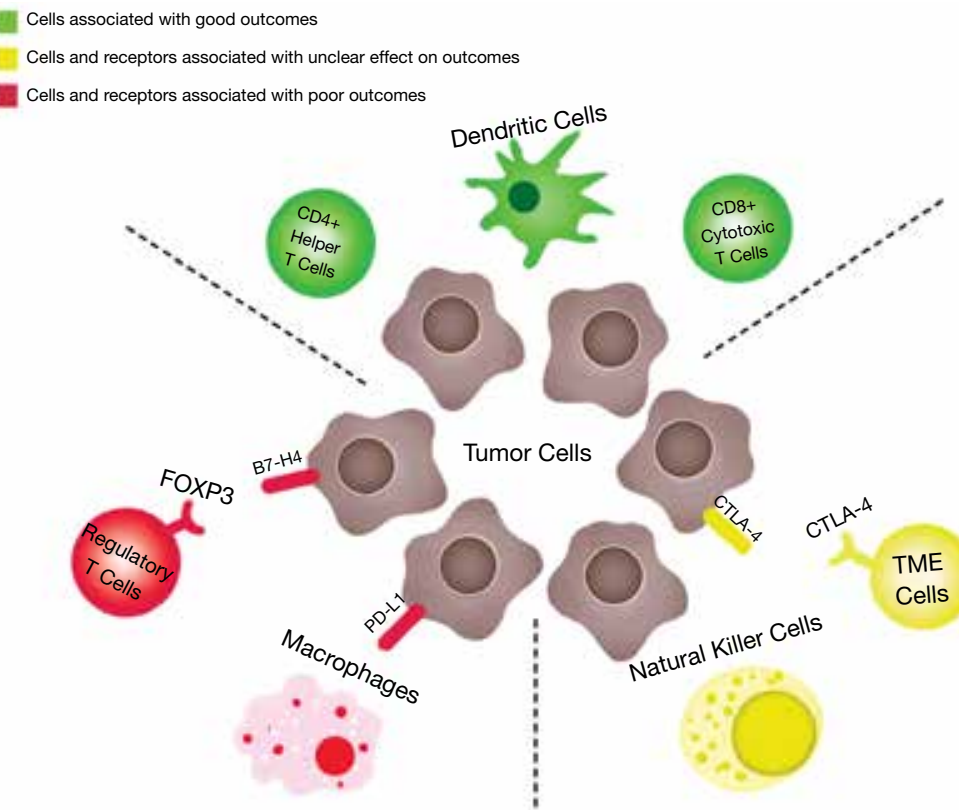

Figure 1 Biliary tract cancer tumor microenvironment. The cells and checkpoints in this schematic have all been described in BTC TME. As per the key-green cells (CD4+ helper T cells, CD8+ cytotoxic T cells, Dendritic cells) have been associated with good outcomes. Yellow cells and checkpoints (Natural Killer cells and CTLA-4) have unclear effect on outcomes. Red cells and checkpoints (Macrophages, FOXP3, B7-H4, PD-L1) have been associated with poor outcomes.

ECC patients have a significantly poorer prognosis (33). Interestingly in one study high CTLA-4 expression had correlation with both improved disease free interval and OS in hilar BTC (34). Multivariate analysis in another study reported that CD4+ TILs, PD-L1 expression and $\mathrm{N}$-cadherin expression were independent prognostic factors for overall survival [hazard ratio $(\mathrm{HR})=0.61 ; 95 \%$ confidence interval (CI), 0.38-1.00; $\mathrm{HR}=4.27 ; 95 \% \mathrm{CI}$, 1.82-9.39; HR =2.20; 95\% CI, 1.18-3.92, respectively) (35).

Gene expression of checkpoints has also been studied in BTC. In a study of over 260 molecularly characterized tumors the worst prognosis was in BTC patients with hypermutated tumors and elevated gene expression of checkpoint molecules including CTLA-4 and PDL-1. Almost half of cases (45\%) showed increased expression of immune checkpoint molecules [CTLA4, IDO1, HAVCR2, TNFRSF9, BTLA, CD274 (PD-L1), PDCD1, LAG3, and TNFRSF4] (36). Overall, these studies suggest that checkpoints may be actively suppressing the host immune response in patients with BTC and can be a potential target for future therapies, providing a rationale for clinical trials to assess the efficacy and safety of anti-PD-1 or PD-L1 therapies. Figure 1 is a schematic summarizing the immune tumor microenvironment (TME) in BTC including immune cells and checkpoints that have been described in the literature.

\section{Mismatch repair, microsatellite status and TMB in BTC}

Two well-known molecular subsets of cancers that have excellent response to ICIs include tumors with increased tumor mutation burden (TMB) and deficiency in mismatch repair (dMMR) proteins or high-level microsatellite instability (MSI-H). In a comprehensive genomic report that molecularly characterized 260 BTC tumors, $5.9 \%$ were considered hypermutated (36). A recent study molecularly profiled 1,502 BTC samples of which 352 had TMB profiled. A total of $14 / 352$ or $4 \%$ were considered TMB high based on a cutoff of 17 somatic missense mutations per megabase of targeted sequence ( $7 / 198$ or $3.5 \%$ for ICC, $1 / 50$ or $2 \%$ for ECC, $6 / 104$ or $5.8 \%$ for GBC) (37). These studies suggest BTC usually has low TMB but there have been reports of ICC cases with higher mutational load responding well to ICI $(38,39)$.

Several studies have sought to establish the overall 
Table 1 Single agent ICI studies with reported outcomes in BTC

\begin{tabular}{|c|c|c|c|c|c|}
\hline $\begin{array}{l}\text { Study } \\
\text { design/setting }\end{array}$ & Treatment arms & $\begin{array}{l}\text { Checkpoint } \\
\text { target(s) }\end{array}$ & Number of patients & Outcomes & Refs \\
\hline Phase lb & Pembrolizumab & PD-1 & 24 (all PD-L1 $\geq 1 \%$ ) & $\begin{array}{l}13 \% \mathrm{PR}, 17 \% \mathrm{SD} \text {, mPFS } 1.8 \text { mos, mOS } \\
6.2 \text { mos, long time responders }>40 \text { weeks } \\
\text { reported }\end{array}$ & $(45)$ \\
\hline Phase II & Pembrolizumab & PD-1 & 104 (61 PD-L1 CPS $\geq 1)$ & $\begin{array}{l}5.8 \% \text { PR ( } 6.6 \% \text { PDL } 1+, 2.9 \% \text { PDL } 1-) \\
\text { mPFS } 2.0 \text { mos, mOS } 7.4 \text { mos }\end{array}$ & $(45)$ \\
\hline Phase II & Nivolumab & PD-1 & $\begin{array}{l}54(63 \% \text { ICC, } 11 \% \text { ECC, } 26 \% \\
\text { GBC) }\end{array}$ & $\begin{array}{l}22 \% \text { PR, } 60 \% \text { DCR, mPFS } 4 \text { mos, mOS } \\
14.2 \text { mos }\end{array}$ & $(47)$ \\
\hline $\begin{array}{l}\text { Phase II/Asian } \\
\text { cohort }\end{array}$ & Durvalumab & PD-L1 & $\begin{array}{l}42 \text { ( } 31 \% \text { ICC, } 19 \% \text { ECC, } 45 \% \\
\text { GBC); (59\% PD-L1+ } \geq 1 \% 14 \%, \\
\text { PD-L1+ } \geq 25 \%)\end{array}$ & $4.8 \%$ PR, mPFS 1.5 mos, mOS 8.1 mos & $(48)$ \\
\hline
\end{tabular}

These trials enrolled patients after failure of at least one standard treatment regimen for advanced BTC or inability to receive standard treatment. PR, partial response; SD, stable disease; CPS, combined positive score; mos, months; mPFS, median progression-free survival; mOS, median overall survival; DCR, disease control rate; ORR, objective response rate.

incidence of $\mathrm{dMMR}$ or MSI in BTC. In one study, $5 \%$ of GBC, $5-13 \%$ of ECC, $10 \%$ of ICC and ampullary carcinomas were dMMR or MSI-H (40). In another study 91 cholangiocarcinoma samples were analyzed (24 ICC, 67 ECC) by immunohistochemistry and dMMR status was found in 20 tumors (22\%) (41). In another study, 102 specimens were examined [35 ICC, 67 ECC (42 extrahepatic and 25 distal)] for dMMR via immunohistochemistry and for MSI status by PCR. Only $1 \%$ had dMMR but the study did not include aggressive samples (42). Finally, the Weinberg et al. study reported $7 / 352$ or $2 \%$ of samples had MSI-H status (5/198 ICC, 1/50 ECC, 1/104 GBC) (37).

In the dMMR or MSI-H subsets of BTC, ICI therapy has shown success. In a seminal study of pembrolizumab across 12 tumor types with $\mathrm{dMMR}, 4$ BTC cases were included. For these patients a $100 \%$ disease control rate (DCR) was reported including one patient achieving a complete response (CR) and three achieving stable disease (SD) (43). A case study of a previously treated ECC patient with MSI high status achieved a partial response (PR) and then SD for 13 months following the start of pembrolizumab (44). Overall a minority of BTC patients have $\mathrm{dMMR}$ or MSI-H status, but in those that do, benefit from single agent ICI therapy can be substantial and we recommend all BTC patients be assessed for MMR deficiency—a recommendation consistent with current NCCN guidelines.

\section{Single agent ICI trials}

Multiple small studies have assessed the potential benefit of single agent ICI therapy in BTC. Abstracts and published articles with reported outcomes with single agent ICI are listed in Table 1. These trials enrolled patients after failure of at least one standard treatment regimen for advanced BTC or inability to receive standard treatment. Most, if not all patients, had proficient MMR status and microsatellite stable status in these trials unless mentioned. A phase IB trial with pembrolizumab (Keynote 028), enrolling only patients with positive PD-L1 tumor expression (PDL1 $\geq 1 \%$ ), reported a $13 \%$ ORR among 24 BTC patients and suggested preliminary activity of ICI in BTC (45). This trial had a mix of BTC subtypes including ICC and ECC. Unfortunately, the larger confirmatory study of pembrolizumab (Keynote 158) in 104 patients with advanced BTC (both $\mathrm{PD}-\mathrm{L} 1$ positive and negative patients were included) reported an ORR of $5.8 \%$ with $16 \%$ of patients experiencing SD. It should be noted that 2 patients in this study had a duration of response (DOR) greater than 15 months. PD-L1 status did not correlate with outcomes in the study (45).

The results of a non-randomized phase I study of nivolumab alone or in combination with cisplatin plus gemcitabine in a Japanese cohort has been published. In the 
Table 2 ICI combination studies with reported outcomes in BTC

\begin{tabular}{|c|c|c|c|c|c|}
\hline Study design/setting & Treatment arms & Checkpoint target(s) & Number of patients & Outcomes & Refs \\
\hline $\begin{array}{l}\text { Phase I/Japanese cohort } \\
\text { (chemotherapy naïve } \\
\text { cohort) }\end{array}$ & $\begin{array}{l}\text { Nivolumab + } \\
\text { cisplatin + } \\
\text { gemcitabine }\end{array}$ & PD-1 & $\begin{array}{l}34 \text { (50\% ICC, 17\% ECC, } \\
33 \% \mathrm{GBC})\end{array}$ & $\begin{array}{l}37 \% \text { PR, mPFS } 4.2 \text { mos, } \\
\text { mOS } 15.4 \text { mos }\end{array}$ & $(46)$ \\
\hline Phase I & $\begin{array}{l}\text { Tremelimumab + } \\
\text { RFA }\end{array}$ & CTLA-4 & 16 & $\begin{array}{l}12.5 \% \text { PR, mPFS } 3.4 \text { mos, } \\
\text { mOS } 6.0 \text { mos }\end{array}$ & (53) \\
\hline Phase lb & $\begin{array}{l}\text { Pembrolizumab + } \\
\text { ramucirumab }\end{array}$ & PD-1 & 26 (ECC 25\%, ICC 42\%) & $\begin{array}{l}\text { 4\% PR, mPFS } 1.6 \text { mos, } \\
\text { mOS } 6.4 \text { mos }\end{array}$ & (54) \\
\hline
\end{tabular}

These trials enrolled patients after failure of at least one standard treatment regimen for advanced BTC unless noted. PR, partial response; $\mathrm{SD}$, stable disease; CPS, combined positive score; mos, months; mPFS, median progression-free survival; mOS, median overall survival; $\mathrm{DCR}$, disease control rate; ORR, objective response rate; $\mathrm{mDOR}$, median duration of response; TMB, tumor mutation burden.

monotherapy cohort there were no CR and there was one $\mathrm{PR}$ in 30 patients. The partial responder was noted to have hereditary nonpolyposis colorectal cancer (dMMR status) and had a PFS of 14.1 months and OS of 19.2 months. Median OS was longer in the patients with PD-L1 positive tumor cells ( $\mathrm{n}=5$, mOS 11.6 months) than PD-L1 negative patients ( $\mathrm{n}=23, \mathrm{mOS} 5.2$ months) in this trial (46). Another phase II study of nivolumab in advanced BTC patients $(\mathrm{n}=54)$ who progressed after at least one line reported a $22 \% \mathrm{PR}$ rate and $38 \% \mathrm{SD}$ rate with a combined DCR of $60 \%$. For evaluable patients mOS was 14.22 months and durable responses over 1 year were seen (47). This phase II study with relatively promising results had $63 \%$ ICC patients and was in an American population. A phase I study of durvalumab, an anti-PD-L1 therapy, alone or in combination with tremelimumab was reported in an Asian cohort. In both the single and combination cohorts the median number of prior chemotherapy regimens was 2 . The monotherapy arm with durvalumab was tolerated well and reported a PR in $2 / 42$ patients (5\%), DCR of $16.7 \%$ at 12 weeks and mOS of 8.1 months (48). Treatment-related adverse events (TRAEs) with single ICI were tolerable in the studies mentioned above. Grade 3-4 events were reported in $13.5 \%$ and $16.7 \%$ of patients in the two pembrolizumab studies, $20 \%$ and $13 \%$ in the two nivolumab studies and $19 \%$ in the durvalumab study.
Novel ICI therapy is being explored. M7824 (MSB0011359C) is an innovative first-in-class bifunctional fusion protein composed of 2 extracellular domains of TGF-beta receptor II (a TGF- $\beta$ "trap") fused to a human IgG1 monoclonal antibody specific PD-L1. Based on preclinical data that tumors with poor anti-PD-L1 or anti-PD-1 response have increased TGF- $\beta$ signaling, this bifunctional protein has dual function to both block PDL1 and sequester TGF- $\beta$ molecules (51). A phase II study in an Asian population reported durable responses in $8 / 30$ patients $(27 \%)$. TRAEs occurred in $60 \%$ of patients; the most common TRAEs were maculopapular rash and pyrexia (13.3\% each). Ten patients (33.3\%) experienced grade 3 or higher TRAEs, including 3 deaths (2 from interstitial lung disease) (49).

\section{ICI combination trials}

Combining ICIs with other therapies including additional immunotherapies, chemotherapy, local therapies and targeted therapies have all been strategies to increase response rates and improve outcomes. Abstracts and published articles with reported outcomes of combination studies are listed in Table 2.

The combination of durvalumab and tremelimumab, a fully human monoclonal antibody that binds to CTLA- 
Table 3 Ongoing or new trials with ICI in BTC

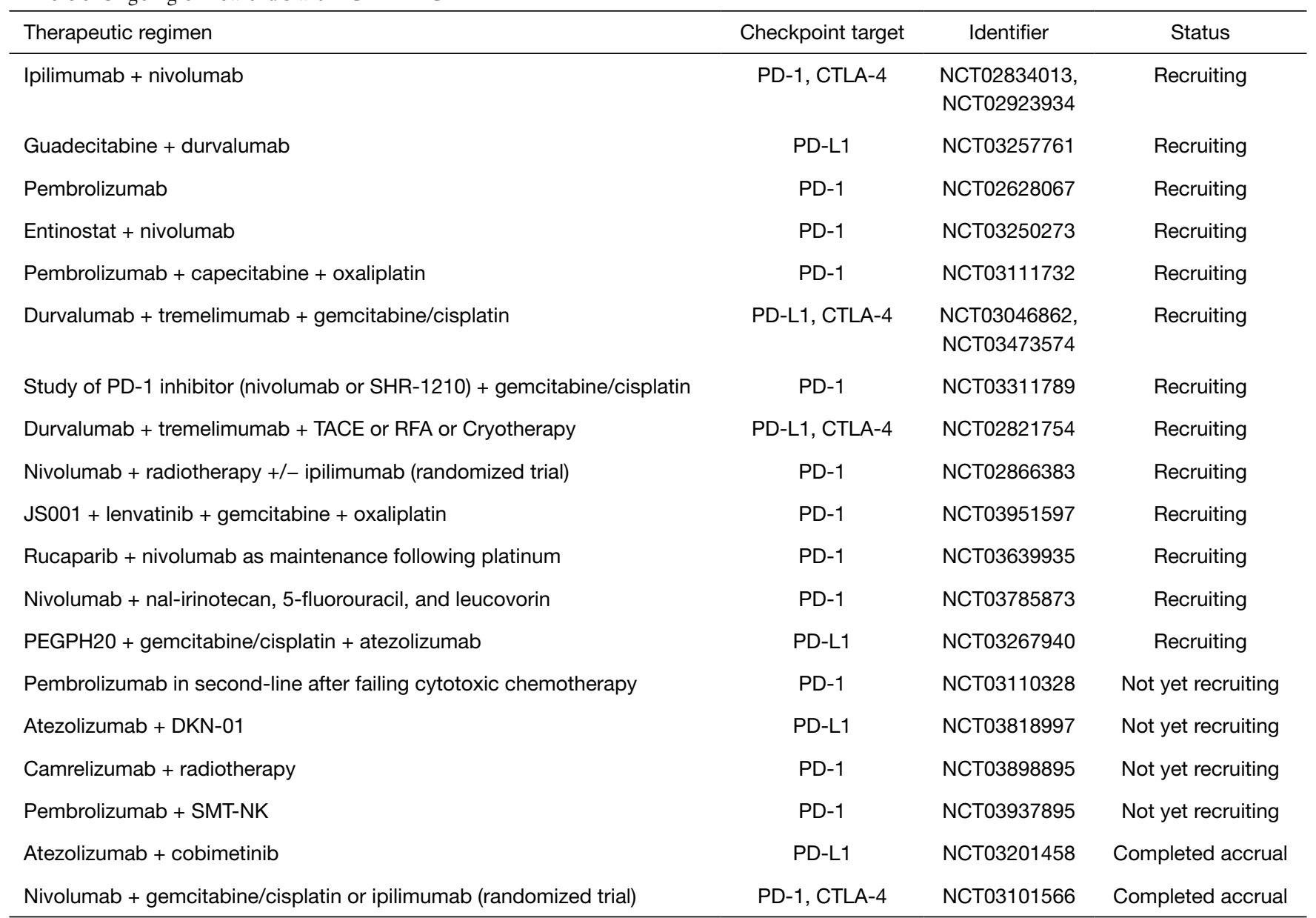

4 resulting in inhibition of B7-CTLA-4-mediated downregulation of T-cell activity, was tolerated well with $23 \%$ of patients experiencing grade 3 or 4 AEs. Reported outcomes included a PR in $11 \%$ of patients (7/65), DCR of $32.2 \%$ at 12 weeks and mOS of 10.1 months (48). GM$\mathrm{CSF}$ is a cytokine growth factor which may promote CD8+ $\mathrm{T}$ cell infiltration into tumors and tumor antigen-specific $\mathrm{T}$ cell expansion (56). Kelley et al. reported compelling early results of a 27-patient trial of pembrolizumab combined with GM-CSF in BTC patients that progressed following standard therapy. It included 19 ICC, 7 ECC and 1 patient with mixed subtype. Preliminary data reported a PR in 5/24 patients $(21 \%)$ and minor regression and Ca19-9 decline in two additional patients. Final PFS and OS analyses for the expanded cohort are expected soon. Reported Grade 3 or 4 $\mathrm{AE}$ was tolerable at $15 \%$ (52).

Preclinical studies have suggested that chemotherapy (including gemcitabine and 5-FU) can upregulate checkpoint expression and change immune cell infiltrate $(57,58)$. Ueno et al. reported a $37 \% \mathrm{PR}$ rate in a chemotherapy-naïve Japanese cohort that received nivolumab plus gemcitabine and cisplatin. This is an improvement in comparison to the historical response rate reported in the ABC-02 trial of $26 \%$ with gemcitabine and cisplatin alone, with the significant caveat that it was a nonrandomized study in a different ethnic population. Median OS was 15.4 months and mPFS was 4.2 months in this study which can be compared to 11.3 months mOS and 8 months mPFS with combination chemotherapy alone in the ABC02 trial $(9,46)$. NCT03101566 is a similar phase II study combining nivolumab with gemcitabine and cisplatin in an American population that has completed accrual but has not reported outcomes of yet. Multiple studies combining ICI and chemotherapy are ongoing and are listed in Table 3.

Another combination strategy includes combining local therapies with ICI. Tremelimumab with local interventions 
including transcatheter arterial chemoembolization (TACE), radiofrequency (RFA), or cryoablation (CA) in patients with hepatocellular carcinoma or ICC is the focus of two trials (NCT01853618, NCT02821754). The rationale behind this trial came from pre-clinical studies in hepatocellular carcinoma and BTC which showed that tremelimumab induces a peripheral immune and $\mathrm{T}$ cell response which may enhance the effect of the combined therapy approach locally and systemically $(59,60)$. Results of the NCT01853618 trial have been reported including that the combination appears safe and leads to the accumulation of intratumoral CD8+ $\mathrm{T}$ cells as well as activation of $\mathrm{T}$ cells in the peripheral blood of responding patients $(53,61)$. Clinical activity of tremelimumab in combination with microwave ablation in BTC patients who had progressed after at least one line of chemotherapy has been reported. Of 16 patients evaluable 2 had a PR, 5 achieved SD and mPFS and mOS was 3.4 and 6.0 months, respectively (53). NCT01853618 is no longer recruiting patients but NCT02821754 is actively recruiting.

The combination of the monoclonal antibody ramucirumab which targets vascular endothelial growth factor (VEGF) receptor 2 plus pembrolizumab was explored in a phase I trial in patients with previously treated advanced or metastatic BTC based on preclinical data suggesting VEGF molecules may regulate checkpoint expression on dendritic cells (62). Unfortunately, reported mPFS and mOS with this combination was 1.6 and 6.4 months (54). Another study combined the multitargeted tyrosine kinase inhibitor of VEGF receptors lenvatinib with PD-1 blockade (pembrolizumab or nivolumab) in 14 ICC patients who had received at least 2 prior anticancer therapies. It has reported promising preliminary results including a $21 \%$ ORR and 93\% DCR (55).

Epigenetic modulating drugs (EMD) are capable of altering the immunogenicity of the TME. Entinostat, a histone deacetylase inhibitor (HDACi), has been shown to have immune modulatory effects including increasing tumor cell expression of MHC II and decreasing regulatory $\mathrm{T}$ cell and myeloid-derived suppressor cell function in mouse models (63). DNMT (DNA methyltransferase) inhibitors, another EMD, have been shown to increase tumor associated antigens, co-stimulatory molecule expression and immune checkpoint expression in cancer cell lines $(64,65)$. EMD and anti-PD-1 combination therapy in animal models have shown synergy (66). Currently there is a phase II trial investigating entinostat with nivolumab (NCT03250273) as well as a study combining the DNMT inhibitor guadecitabine with durvalumab (NCT03257761) with results expected in 2020 (67).

Up to $25 \%$ of BTC patients have molecular alterations in DNA repair genes, and ongoing and future trials of DNA repair modulators with ICIs in BTC are an important area of investigation (68). A phase II study is using the PARP inhibitor rucaparib in combination with nivolumab as a maintenance strategy in BTC patients who have received first line platinum based chemotherapy and have not had progression after 4-6 months (69). Additional studies combining ICI and targeted therapies in BTC are listed in Table 3.

\section{Conclusions}

The BTC TME including tumor specific adaptive and innate immune cells and checkpoint expression has been correlated with clinical outcomes and prognosis. The incidence of dMMR or MSI status in BTC appears to be rare but given robust activity of ICI in these subtypes, all BTC should be tested for MMR or MSI status. Single agent PD-1 or PD-L1 inhibition is well tolerated and efficacy data varied between studies with reported ORR between $3-22 \%$ and mOS range of 5.2-14.2 months. These studies contained patients that had progressed after at least one line of chemotherapy for advanced disease. In one study PDL1 positive patients had improved outcomes (Ueno et al.) and in another study an American based cohort (Kim et al.) appeared to have higher response rates and longer survival compared to Japanese and Asian cohorts (Ueno et al. and Ioka et al.). Activity in certain patient populations and tolerable side effect profiles warrants the exploration of potential patient biomarkers to stratify patients into who is likely and unlikely to respond. Pooled subanalyses of responses and outcomes by anatomic subsite (ICC $v s$. ECC vs. GBC) could also be studied. Overall these results suggest that more studies are warranted to possibly establish single agent anti-PD1 or PD-L1 therapy as a second line option in BTC. As far as combination studies, the addition of nivolumab to gemcitabine and cisplatin in the first line appeared to increase response rates and OS in an Asian population (Ueno et al.). Results from a similar study will be presented soon but this chemoimmunotherapy strategy should be considered for evaluation in a phase III randomized study to further discern the impact of adding ICI to chemotherapy. Additional combinations have promising preliminary activity data including pembrolizumab plus GM-CSF and pembrolizumab plus lenvatinib. Overall the benefit from immunotherapy and 
ICI in BTC is still unclear and additional results from multiple studies are expected soon.

\section{Acknowledgments}

We thank Kimberly Cheung (Graphic Designer, New York, NY, USA) for assisting with the figure illustration.

\section{Footnote}

Conflicts of Interest: The authors have no conflicts of interest to declare.

Ethical Statement: The authors are accountable for all aspects of the work in ensuring that questions related to the accuracy or integrity of any part of the work are appropriately investigated and resolved.

\section{References}

1. Patel T. Increasing incidence and mortality of primary intrahepatic cholangiocarcinoma in the United States. Hepatology 2001;33:1353-7.

2. Shaib Y, El-Serag HB. The Epidemiology of Cholangiocarcinoma. Semin Liver Dis 2004;24:115-25.

3. Ghouri YA, Mian I, Blechacz B. Cancer review: Cholangiocarcinoma. J Carcinog 2015;14:1.

4. Siegel RL, Miller KD, Jemal A. Cancer statistics, 2018. CA Cancer J Clin 2018;68:7-30.

5. de Groen PC, Gores GJ, LaRusso NF, et al. Biliary Tract Cancers. N Engl J Med 1999;341:1368-78.

6. DeOliveira ML, Cunningham S, Cameron J, et al. Cholangiocarcinoma: Thirty-one-Year Experience With 564 Patients at a Single Institution. Ann Surg 2007;245:755-62.

7. Park J, Kim MH, Kim K, et al. Natural History and Prognostic Factors of Advanced Cholangiocarcinoma without Surgery, Chemotherapy, or Radiotherapy: A LargeScale Observational Study. Gut Liver 2009;3:298-305.

8. Spolverato G, Bagante F, Weiss M, et al. Comparative performances of the 7th and the 8th editions of the American Joint Committee on Cancer staging systems for intrahepatic cholangiocarcinoma. J Surg Oncol 2017;115:696-703.

9. Valle J, Wasan H, Palmer DH, et al. Cisplatin plus Gemcitabine versus Gemcitabine for Biliary Tract Cancer. N Engl J Med 2010;362:1273-81.

10. Lamarca A, Palmer DH, Wasan HS, et al. ABC-06 I A randomised phase III, multi-centre, open-label study of active symptom control (ASC) alone or ASC with
oxaliplatin/5-FU chemotherapy (ASC+mFOLFOX) for patients (pts) with locally advanced / metastatic biliary tract cancers (ABC) previously-treated with cisplatin/ gemcitabine (CisGem) chemotherapy. JCO 2019;37:4003.

11. Ebata T, Ercolani G, Alvaro D, et al. Current Status on Cholangiocarcinoma and Gallbladder Cancer. Liver Cancer 2016;6:59-65.

12. Jain A, Kwong LN, Javle M. Genomic Profiling of Biliary Tract Cancers and Implications for Clinical Practice. Curr Treat Options Oncol 2016;17:58.

13. Merla A, Liu KG, Rajdev L. Targeted Therapy in Biliary Tract Cancers. Curr Treat Options Oncol 2015;16:48.

14. Lowery MA, Abou-Alfa GK, Burris HA, et al. Phase I study of AG-120, an IDH1 mutant enzyme inhibitor: Results from the cholangiocarcinoma dose escalation and expansion cohorts. JCO 2017;35:4015.

15. Bekaii-Saab TS, Valle JW, Borad MJ, et al. Trial design for a phase 3 study evaluating pemigatinib (INCB054828) versus gemcitabine plus cisplatin chemotherapy in firstline treatment of patients with cholangiocarcinoma with FGFR2 rearrangement. JCO 2019;37:TPS462.

16. El-Serag HB, Engels EA, Landgren O, et al. Risk of hepatobiliary and pancreatic cancers after hepatitis $\mathrm{C}$ virus infection: A population-based study of U.S. veterans. Hepatology 2009;49:116-23.

17. Augustine MM, Fong Y. Epidemiology and Risk Factors of Biliary Tract and Primary Liver Tumors. Surg Oncol Clin N Am 2014;23:171-88.

18. Marks EI, Yee NS. Immunotherapeutic approaches in biliary tract carcinoma: Current status and emerging strategies. World J Gastrointest Oncol 2015;7:338-46.

19. Goeppert B, Frauenschuh L, Zucknick M, et al. Prognostic impact of tumour-infiltrating immune cells on biliary tract cancer. Br J Cancer 2013;109:2665-74.

20. Nakakubo Y, Miyamoto M, Cho Y, et al. Clinical significance of immune cell infiltration within gallbladder cancer. Br J Cancer 2003;89:1736-42.

21. Oshikiri T, Miyamoto M, Shichinohe T, et al. Prognostic value of intratumoral CD8+ T lymphocyte in extrahepatic bile duct carcinoma as essential immune response. J Surg Oncol 2003;84:224-8.

22. Takagi S. Dendritic cells, T-cell infiltration, and grp94 expression in cholangiocellular carcinoma. Hum Pathol 2004;35:881-6.

23. Kitano Y, Okabe H, Yamashita Y, et al. Tumourinfiltrating inflammatory and immune cells in patients with extrahepatic cholangiocarcinoma. Br J Cancer 2018;118:171-80. 
24. McNamara MG, Templeton AJ, Maganti M, et al. Neutrophil/lymphocyte ratio as a prognostic factor in biliary tract cancer. Eur J Cancer 2014;50:1581-9.

25. Lin G, Liu Y, Li S, et al. Elevated neutrophil-tolymphocyte ratio is an independent poor prognostic factor in patients with intrahepatic cholangiocarcinoma. Oncotarget 2016;7:50963-71.

26. Suleiman Y, Coppola D, Zibadi S, et al. Prognostic value of tumor-infiltrating lymphocytes (TILs) and expression of PD-L1 in cholangiocarcinoma. JCO 2015;33:294.

27. Sabbatino F, Villani V, Yearley JH, et al. PD-L1 and HLA Class I Antigen Expression and Clinical Course of the Disease in Intrahepatic Cholangiocarcinoma. Clin Cancer Res 2016;22:470-8.

28. Ye Y, Zhou L, Xie X, et al. Interaction of B7-H1 on intrahepatic cholangiocarcinoma cells with PD-1 on tumor-infiltrating $\mathrm{T}$ cells as a mechanism of immune evasion. J Surg Oncol 2009;100:500-4.

29. Fontugne J, Augustin J, Pujals A, et al. PD-L1 expression in perihilar and intrahepatic cholangiocarcinoma.

Oncotarget 2017;8:24644-51.

30. Gani F, Nagarajan N, Kim Y, et al. Program Death 1 Immune Checkpoint and Tumor Microenvironment: Implications for Patients With Intrahepatic Cholangiocarcinoma. Ann Surg Oncol 2016;23:2610-7.

31. Lim YJ, Koh J, Kim K, et al. High ratio of programmed cell death protein $1(\mathrm{PD}-1)(+) / \mathrm{CD} 8(+)$ tumor-infiltrating lymphocytes identifies a poor prognostic subset of extrahepatic bile duct cancer undergoing surgery plus adjuvant chemoradiotherapy. Radiother Oncol 2015;117:165-70.

32. Ha H, Nam AR, Bang JH, et al. Soluble programmed death-ligand 1 (sPDL1) and neutrophil-to-lymphocyte ratio (NLR) predicts survival in advanced biliary tract cancer patients treated with palliative chemotherapy. Oncotarget 2016;7:76604-12.

33. Wada S. Clinicopathological implications of newly defined immune checkpoints in extra-hepatic cholangiocarcinoma. JCO 2015;33:e15151.

34. Lim YJ, Koh J, Kim K, et al. Clinical Implications of Cytotoxic T Lymphocyte Antigen-4 Expression on Tumor Cells and Tumor-Infiltrating Lymphocytes in Extrahepatic Bile Duct Cancer Patients Undergoing Surgery Plus Adjuvant Chemoradiotherapy. Target Oncol 2017;12:211-8.

35. Ueno T, Tsuchikawa T, Hatanaka KC, et al. Prognostic impact of programmed cell death ligand 1 (PD-L1) expression and its association with epithelial-mesenchymal transition in extrahepatic cholangiocarcinoma. Oncotarget
2018;9:20034-47.

36. Nakamura H, Arai Y, Totoki Y, et al. Genomic spectra of biliary tract cancer. Nat Genet 2015;47:1003-10.

37. Weinberg BA, Xiu J, Lindberg MR, et al. Molecular profiling of biliary cancers reveals distinct molecular alterations and potential therapeutic targets. J Gastrointest Oncol 2019;10:652-62.

38. Mou H, Yu L, Liao Q, et al. Successful response to the combination of immunotherapy and chemotherapy in cholangiocarcinoma with high tumour mutational burden and PD-L1 expression: a case report. BMC Cancer 2018;18:1105.

39. Sui M, Li Y, Wang H, et al. Two cases of intrahepatic cholangiocellular carcinoma with high insertiondeletion ratios that achieved a complete response following chemotherapy combined with PD-1 blockade. J Immunother Cancer 2019;7:125.

40. Silva VW, Askan G, Daniel TD, et al. Biliary carcinomas: pathology and the role of DNA mismatch repair deficiency. Chin Clin Oncol 2016;5:62.

41. Kunk PR, Obeid JM, Winters K, et al. Mismatch repair deficiency in cholangiocarcinoma. JCO 2018;36:269.

42. Winkelmann R, Schneider M, Hartmann S, et al. Microsatellite Instability Occurs Rarely in Patients with Cholangiocarcinoma: A Retrospective Study from a German Tertiary Care Hospital. Int J Mol Sci 2018;19:1421.

43. Le DT, Durham JN, Smith KN, et al. Mismatch repair deficiency predicts response of solid tumors to PD-1 blockade. Science 2017;357:409-13.

44. Czink E, Kloor M, Goeppert B, et al. Successful immune checkpoint blockade in a patient with advanced stage microsatellite-unstable biliary tract cancer. Cold Spring Harb Mol Case Stud 2017;3:a001974.

45. Bang YJ, Ueno M, Malka D, et al. Pembrolizumab (pembro) for advanced biliary adenocarcinoma: Results from the KEYNOTE-028 (KN028) and KEYNOTE-158 (KN158) basket studies. JCO 2019;37:4079.

46. Ueno M, Ikeda M, Morizane C, et al. Nivolumab alone or in combination with cisplatin plus gemcitabine in Japanese patients with unresectable or recurrent biliary tract cancer: a non-randomised, multicentre, open-label, phase 1 study. The Lancet Gastroenterology \& Hepatology [Internet]. 2019 May 17 [cited 2019 May 27]. Available online: http://www.sciencedirect.com/science/article/pii/ S246812531930086X

47. Kim RD, Kim DW, Alese OB, et al. A phase II study of nivolumab in patients with advanced refractory biliary tract cancers (BTC). JCO 2019;37:4097. 
48. Ioka T, Ueno M, Oh DY, et al. Evaluation of safety and tolerability of durvalumab (D) with or without tremelimumab (T) in patients (pts) with biliary tract cancer (BTC). JCO 2019;37:387.

49. Yoo C, Oh DY, Choi HJ, et al. 153OM7824 (MSB0011359C), a bifunctional fusion protein targeting transforming growth factor $\beta$ (TGF- $\beta$ ) and PD-L1, in Asian patients with pretreated biliary tract cancer (BTC): Efficacy by BTC subtype. Ann Oncol [Internet]. 2018 Nov 1 [cited 2019 May 27];29(suppl_9). Available online: https://academic.oup.com/annonc/article/29/suppl_9/ mdy432.005/5200092

50. Fujiwara Y, Koyama T, Helwig C, et al. M7824 (MSB0011359C), a bifunctional fusion protein targeting PD-L1 and TGF- $\beta$, in Asian patients with advanced solid tumors. JCO 2018;36:762.

51. Gatti-Mays ME, Gulley JL. M7824: A promising new strategy to combat cancer immune evasion. Oncoscience 2018;5:269-70.

52. Kelley RK, Mitchell E, Behr S, et al. Phase II trial of pembrolizumab (PEM) plus granulocyte macrophage colony stimulating factor (GM-CSF) in advanced biliary cancers (ABC). JCO 2018;36:386.

53. Xie C, Duffy AG, Mabry-Hrones D, et al. Tremelimumab in Combination With Microwave Ablation in Patients With Refractory Biliary Tract Cancer. Hepatology 2019;69:2048-60.

54. Arkenau HT, Martin-Liberal J, Calvo E, et al. Ramucirumab Plus Pembrolizumab in Patients with Previously Treated Advanced or Metastatic Biliary Tract Cancer: Nonrandomized, Open-Label, Phase I Trial (JVDF). Oncologist 2018;23:1407-e136.

55. Lin J, Shi W, Zhao S, et al. Lenvatinib plus checkpoint inhibitors in patients (pts) with advanced intrahepatic cholangiocarcinoma (ICC): Preliminary data and correlation with next-generation sequencing. JCO 2018;36:500.

56. Wei XX, Chan S, Kwek S, et al. Systemic GMCSF Recruits Effector T Cells into the Tumor Microenvironment in Localized Prostate Cancer. Cancer Immunology Research 2016;4:948-58.

57. Koido S, Kan S, Yoshida K, et al. Immunogenic modulation of cholangiocarcinoma cells by chemoimmunotherapy. Anticancer Res 2014;34:6353-61.

58. Liu WM, Fowler DW, Smith P, et al. Pre-treatment with chemotherapy can enhance the antigenicity and immunogenicity of tumours by promoting adaptive immune responses. Br J Cancer 2010;102:115-23.

59. Agdashian D, ElGindi M, Xie C, et al. The effect of anti-
CTLA4 treatment on peripheral and intra-tumoral T cells in patients with hepatocellular carcinoma. Cancer Immunol Immunother 2019;68:599-608.

60. Duffy AG, Ulahannan SV, Makorova-Rusher O, et al. Tremelimumab in combination with ablation in patients with advanced hepatocellular carcinoma. J Hepatol 2017;66:545-51.

61. Duffy AG, Makarova-Rusher OV, Pratt D, et al. Tremelimumab: A monoclonal antibody against CTLA4-In combination with subtotal ablation (trans catheter arterial chemoembolization (TACE), radiofrequency ablation (RFA) or cryoablation) in patients with hepatocellular carcinoma (HCC) and biliary tract carcinoma (BTC). JCO 2016;34:4073.

62. Motz GT, Coukos G. The parallel lives of angiogenesis and immunosuppression: cancer and other tales. Nat Rev Immunol 2011;11:702-11.

63. Smith HJ, McCaw TR, Londono AI, et al. The antitumor effects of entinostat in ovarian cancer require adaptive immunity. Cancer 2018;124:4657-66.

64. Wrangle J, Wang W, Koch A, et al. Alterations of immune response of non-small cell lung cancer with Azacytidine. Oncotarget 2013;4:2067-79.

65. Li H, Chiappinelli KB, Guzzetta AA, et al. Immune regulation by low doses of the DNA methyltransferase inhibitor 5-azacitidine in common human epithelial cancers. Oncotarget 2014;5:587-98.

66. Mazzone R, Zwergel C, Mai A, et al. Epi-drugs in combination with immunotherapy: a new avenue to improve anticancer efficacy. Clin Epigenetics 2017;9:59.

67. Baretti M, Durham JN, Walker R, et al. Entinostat in combination with nivolumab for patients with advanced cholangiocarcinoma and pancreatic adenocarcinoma. JCO 2018;36:TPS4151.

68. Abdel-Wahab R, Ali SM, Borad MJ, et al. Variations in DNA repair genomic alterations and tumor mutation burden in biliary tract cancer (BTC) subtypes. JCO 2018;36:263.

69. Sahai V, Tran NH, Griffith KA, et al. A multicenter phase II trial of rucaparib in combination with nivolumab as maintenance therapy for patients with advanced biliary tract cancer. JCO 2019;37:TPS4153.

Cite this article as: Jakubowski CD, Azad NS. Immune checkpoint inhibitor therapy in biliary tract cancer (cholangiocarcinoma). Chin Clin Oncol 2020;9(1):2. doi: 10.21037/cco.2019.12.10 